\title{
Association of Perfluorooctanoic Acid (PFOA) and Perfluorooctane Sulfonate (PFOS) with Age of Puberty among Children Living near a Chemical Plant
}

\author{
Maria-Jose Lopez-Espinosa, ${ }^{*,+}$ Tony Fletcher, $^{\dagger}$ Ben Armstrong, ${ }_{+}^{+}$Bernd Genser, ${ }^{\ddagger, \S}$ Ketan Dhatariya," \\ Debapriya Mondal, ${ }^{+}$Alan Ducatman, ${ }^{\perp}$ and Giovanni Leonardi ${ }^{\dagger}$ \\ ${ }^{\dagger}$ Department of Social and Environmental Health Research, London School of Hygiene \& Tropical Medicine, 15-17 Tavistock Place, \\ London WC1H 9SH, United Kingdom \\ ${ }^{\ddagger}$ Mannheim Institute of Public Health, Social and Preventive Medicine, University of Heidelberg, Heidelberg, Germany

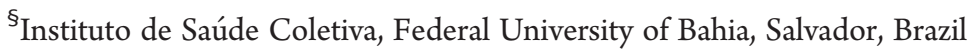 \\ "Elsie Bertram Diabetes Centre, Norfolk and Norwich University Hospital NHS Foundation Trust, Norwich, United Kingdom \\ ${ }^{\perp}$ Department of Community Medicine, West Virginia University School of Medicine, West Virginia, United States
}

ABSTRACT: Animal studies suggest that perfluorocarbons (PFCs) may alter sexual maturation. Relationships of human PFC exposure with puberty are not clear. We conducted a cross-sectional study to investigate whether perfluorooctanoic acid (PFOA) and perfluorooctane sulfonate (PFOS) were associated with indicators of sexual maturation in a 2005-2006 survey of residents with PFOA water contamination from the Mid-Ohio Valley. Participants were 3076 boys and 2931 girls aged $8-18$ years. They were classified as having reached puberty based on either hormone levels (total $>50 \mathrm{ng} / \mathrm{dL}$ and free $>5 \mathrm{pg} / \mathrm{mL}$ testosterone in boys and estradiol $>20 \mathrm{pg} / \mathrm{mL}$ in girls) or onset of menarche. We estimated the odds of having reached puberty classified by these criteria and the fitted median age of reaching puberty in relation to serum PFOA and PFOS concentrations measured when puberty status was assigned. For boys, there was a relationship of reduced odds of reached puberty (raised testosterone) with increasing PFOS (delay of 190 days between the highest and lowest quartile). For girls, higher concentrations of PFOA or PFOS were associated with reduced odds of postmenarche (130 and 138 days of delay, respectively). In conclusion, our study showed a later age of puberty in this population correlated with PFC concentrations.

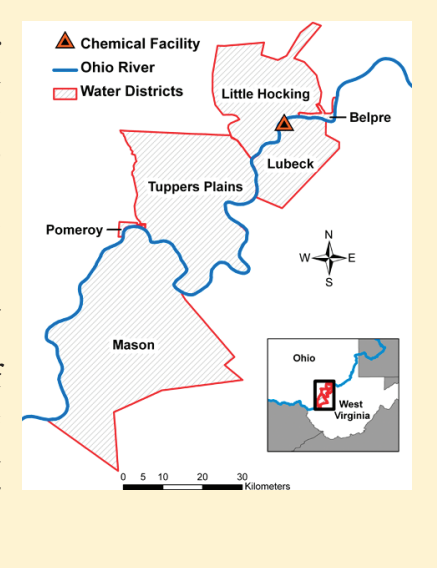

\section{INTRODUCTION}

Perfluorooctanoic acid (PFOA) and perfluorooctane sulfonate (PFOS) are members of the class of man-made perfluorocarbon (PFC) compounds. They are ubiquitously present and persistent in environmental and biological samples. ${ }^{1}$ Concern about the health effects of these compounds has increased due to their reported experimental animal toxicity and potential adverse human health effects. ${ }^{2}$ Several animal studies have highlighted the ability of PFOS and PFOA to affect the endocrine system. ${ }^{3}$ Thus, exposure to higher PFOA concentrations has been associated with lower testosterone levels and higher estradiol levels in male adult rats ${ }^{4,5}$ and with higher progesterone levels in peripubertal female mice. ${ }^{6}$ Exposure to PFOS has been associated with lowered estradiol levels in male monkeys ${ }^{7}$ and elevated testosterone levels in males from some fish species. ${ }^{8}$ Animal studies have also reported the specific effects of PFOA/PFOS treatment during various critical development stages on sexual maturation, although results are inconsistent since one study did not find any association, ${ }^{9}$ while others found a delay $^{10-12}$ or an acceleration ${ }^{6,12,13}$ of pubertal development.

Human studies on potential endocrine effects of PFCs are not consistent. Neither PFOA nor PFOS was associated with estradiol and testosterone levels in American male workers ${ }^{14}$ nor among Danish men. ${ }^{15}$ In a study of adult women in the same community as the present paper, PFOS and PFOA concentrations were positively associated with earlier menopause and PFOS concentrations with lower estradiol levels. ${ }^{16}$ In a study of sexual maturation in a British cohort, prenatal PFOA and PFOS concentrations were not significantly associated with self-reported menarche. ${ }^{17}$ However, there is one American study, available only as an abstract, reporting earlier pubertal maturation (assessed as breast maturation) associated with raised PFOA concentrations. ${ }^{18}$

The underlying mechanism on how PFC compounds may affect the endocrine system remains unclear. A study did not find an estrogen-dependent proliferation capacity for PFOA and PFOS using the human MCF-7 breast cancer cell assay. ${ }^{19}$ However, estrogenic activities of PFOA or PFOS as well as antiestrogenic activities after exposure to a combination of estradiol and PFC have been reported in primary cultured hepatocytes from fish. ${ }^{20}$ The authors concluded that PFC estrogenic effect

Special Issue: Perfluoroalkyl Acid

Received: November 17, 2010

Accepted: April 14, 2011

Revised: April 1, 2011 
may be mediated by the estrogen receptor pathway. ${ }^{20}$ In male rats, it has been reported that the decrease in testosterone levels may be due to reduced conversion of $17 \alpha$-hydroxyprogesterone to androstenedione $e^{5}$ and the increase in serum estradiol levels may be due to aromatase induction in liver ${ }^{4}$ after exposure to PFOA. In addition, an in vitro study has reported PFOA directly inhibits testosterone release from Leydig cells removed from the adult rat testes. ${ }^{4}$

PFOA has been used in the manufacture of fluoropolymers at a chemical plant in Washington, West Virginia since 1951. In 2001, a group of residents, from the Ohio and West Virginia communities in the vicinity of the Washington Works plant, filed a class-action lawsuit, alleging health damage due to contamination of human drinking water supplies with PFOA. The settlement of this lawsuit led to a baseline survey, called the C8 Health Project, conducted during 2005 and 2006 on residents who lived in six contaminated water districts surrounding the chemical plant. $^{21}$ Median PFOA and PFOS serum concentrations in the population presented here were 28.2 and $20.2 \mathrm{ng} / \mathrm{mL}^{21}$ versus 4.2 and $17.5 \mathrm{ng} / \mathrm{mL}$, respectively, in the general American population $^{22}$ tested in samples collected during the same period of time (2005-2006).

The aim of the present study was to assess the associations between PFOA and PFOS concentrations and indicators of sexual maturation (based on levels of sex steroid hormones and self-reported menarche status) in children aged 8-18 years from the Ohio and West Virginia communities, in a cross sectional analysis of data collected during 2005-2006.

\section{MATERIAL AND METHODS}

C8 Health Project and C8 Science Panel Populations. The C8 Health Project enrolled community participants of all ages between August 2005 and July 2006. This community was relatively highly exposed to PFOA; however, PFOS concentrations correspond to US-wide average levels. All participants gave written informed consent prior to inclusion. Enrollment criteria and consent procedures are described in a previous publication. ${ }^{21}$ Briefly, subjects were eligible if they could document public drinking water consumption for at least one year before December 3, 2004, supplied by the contaminated water districts. The C8 Health Project collected data on 69,030 people. The participation rate of people still living in the exposed water districts at survey has been estimated for age groups 5-10, 11-14, and $15-19$ years to be 77,87 , and $95 \%$, respectively. ${ }^{21}$ Within the C8 Health Project population, $69 \%$ further consented to have their contact details provided to the C8 Science Panel to allow participation in further research. Full date of birth is available only for this consented population, which was needed for precise dating of age and pubertal status at time of interview and blood draw. These analyses are restricted to ages 8-18 years when most children reach puberty. There were 4677 boys and 4393 girls aged 8-18 years at enrollment among the 69,030 participants. Of these, 3076 (66\%) boys and 2931 (67\%) girls had available serum biomarkers, PFOA and PFOS concentrations, consent for contact from the C8 Science Panel, accurate information on age of birth (day, month, and year), and questionnaire data. Therefore, the final present study sample comprised 6007 participants.

Serum PFOA and PFOS Determination. Laboratory analyses of PFOA and PFOS were conducted by a commercial laboratory (Exygen, State College, PA, USA). The laboratory analytical methods and quality control procedures were described elsewhere. $^{21}$ Briefly, serum concentrations of PFOA and PFOS were determined using liquid chromatography separation with detection by tandem mass spectrometry. Estimates of precision for PFOA were generally within $\pm 10 \%$ for multiple replicates over the range of $0.5 \mathrm{ng} / \mathrm{mL}$ to $40 \mathrm{ng} / \mathrm{mL}$ with a more precise relative precision measure of approximately $1 \%$ for highly fortified $(10,000 \mathrm{ng} / \mathrm{mL})$ samples. Relative precision estimates for PFOS were similar. The detection limit (LOD) for PFOA and PFOS was $0.5 \mathrm{ng} / \mathrm{mL}$, and observations below LOD were assigned a value of $0.25 \mathrm{ng} / \mathrm{mL}$ (none and $N=11$ in the case of PFOA and PFOS, respectively, for this study population).

Sex Steroid Hormone Determination, Self-Reported Menarche, and Pubertal Status. Clinical laboratory tests were performed at an accredited clinical diagnostic laboratory (LabCorp, Inc., Burlington, NC, USA). Estradiol and total testosterone levels were measured in serum samples by using an electrochemiluminescence immunoassay (ECLIA; Roche Diagnostics, Indianapolis, IN, USA) with LODs of $7 \mathrm{pg} / \mathrm{mL}$ and $10 \mathrm{ng} / \mathrm{dL}$, respectively. Free testosterone was measured by a radioimmunoassay (Coat-a-Count; Siemens Healthcare Diagnostics, Los Angeles, CA, USA) with LOD of $0.2 \mathrm{pg} / \mathrm{mL}$. The number of girls with estradiol levels <LOD was 149, and the number of boys with total testosterone and free testosterone levels <LODs were 158 and 608, respectively.

The participants (or their guardians, for younger participants) completed a questionnaire on sociodemographic characteristics, current and historic residential and employment information, lifestyle, family medical history, and health variables. The questionnaire included a question that was worded as follows: "At what age did you begin menstruation (have your first period)?” Girls were asked to select one of the following categories: $<10$ years, $10-12$ years, $13-15$ years, $\geq 16$ years, have not begun to menstruate, never menstruated, do not know/not sure. Girls who reported not to know/be sure about the beginning of the menstruation were excluded. For statistical analysis, we generated a dichotomous variable: yes (menarche had started) or no (not yet) to be modeled in relation to exact age at interview.

We used cutoff points of $50 \mathrm{ng} / \mathrm{dL}$ and $5 \mathrm{pg} / \mathrm{mL}$ for total and free testosterone in boys and $20 \mathrm{pg} / \mathrm{mL}$ for estradiol in girls as indicators of sexual maturation. These cutoff points have been used in previous studies ${ }^{23-25}$ and performed very well in this population for boys, in discriminating the pre- and postpubertal children. For girls, the sensitivity and specific of the estradiol cutoff was poorer, as is evident in the higher proportions above the cutoff for very young girls and lower proportion below the cutoff for older girls (Table 2). Also, we interpret the fall in the proportion of girls above the cutoff for those $>15$ years to be due to under-reporting of contraceptive use.

The questionnaire provided some information on contraceptive pill usage from responses to an open question on pharmaceutical use, and this was low among older girls. Published data indicate that $9 \%$ of $15-17$ year old girls in the US report oral contraceptive use, ${ }^{26}$ whereas only $2 \%$ reported pill use in this age range in our population. Half of these 36 subjects' estradiol levels were $\leq 20 \mathrm{pg} / \mathrm{mL}$ and thus would have been incorrectly classified as prepubertal, based on estradiol levels alone, although they reported being postmenarchal. We therefore combined information on being postmenarche into the estradiol-based indicator to address the underascertainment of data on hormonal contraceptive use. Thus, the estradiol combined indicator classified girls as postpuberty if either estradiol levels $>20 \mathrm{pg} / \mathrm{mL}$ or they were postmenarchal. Given these 
Table 1. Description of Study Population $(N=6007)$, Mid-Ohio Valley, 2005-2006 ${ }^{a}$

\begin{tabular}{|c|c|c|c|}
\hline variables & categories & boys & girls \\
\hline gender: $\mathrm{N}$ & boys/girls & 3076 & 2931 \\
\hline race: $\mathrm{N}(\%)$ & white & $2964(96.4)$ & $2837(96.8)$ \\
\hline BMI: mean (SD) & $\mathrm{kg} / \mathrm{m}^{2}$ & $22.7(5.6)$ & $22.3(5.5)$ \\
\hline height: mean (SD) & inches & $64.5(7.1)$ & $61.6(5.3)$ \\
\hline \multirow[t]{3}{*}{ household family income: $\mathrm{N}$ (\%) } & $<\$ 20,000 /$ year & $891(36.9)$ & $791(34.4)$ \\
\hline & $\$ 20,000-70,000 /$ year & $1174(48.7)$ & $1187(51.7)$ \\
\hline & $>\$ 70,000 /$ year & $348(14.4)$ & $319(13.9)$ \\
\hline \multirow[t]{3}{*}{ time of sampling: $\mathrm{N}(\%)$} & $<12.00 \mathrm{pm}$ & $1097(35.7)$ & $1031(35.2)$ \\
\hline & $12.00-16.00 \mathrm{pm}$ & $1320(42.9)$ & $1278(43.6)$ \\
\hline & $17.00-19.00 \mathrm{pm}$ & $659(21.4)$ & $622(21.2)$ \\
\hline alcohol consumption: N (\%) & yes & $293(11.4)$ & $324(13.0)$ \\
\hline smoking habit: N (\%) & yes & $126(4.1)$ & $132(4.5)$ \\
\hline \multirow[t]{4}{*}{ PFOA: GM (GSD) } & $\mathrm{Q} 1:<11.4 \mathrm{ng} / \mathrm{mL}$ & $8.4(1.31)$ & $7.7(1.39)$ \\
\hline & $\mathrm{Q} 2: 11.4-23 \mathrm{ng} / \mathrm{mL}$ & $16.5(1.23)$ & $16.3(1.23)$ \\
\hline & Q3:>23-58 ng/mL & $36.4(1.29)$ & $35.7(1.30)$ \\
\hline & Q4:>58 ng/mL & $145.3(1.98)$ & $151.0(1.93)$ \\
\hline \multirow[t]{4}{*}{ PFOS: GM (GSD) } & $\mathrm{Q} 1:<14.3 \mathrm{ng} / \mathrm{mL}$ & $10.2(1.66)$ & $9.8(1.49)$ \\
\hline & $\mathrm{Q} 2: 14.3-19.4 \mathrm{ng} / \mathrm{mL}$ & $16.4(1.09)$ & $16.4(1.09)$ \\
\hline & Q3:>19.4-27 ng/mL & $22.3(1.10)$ & $22.3(1.10)$ \\
\hline & Q4:>27 ng/mL & $36.0(1.29)$ & $35.2(1.26)$ \\
\hline
\end{tabular}

concerns about the predictive value of the estradiol cutoff, we place more emphasis on results using menarche than estradiol as an indicator of pubertal maturation.

Confounders. Covariates considered for inclusion in the models were demographic and lifestyle factors available in the data set as follows: age at survey, body mass index (BMI in quintiles) z-score based on the 2000 CDC growth charts of BMIfor-age, ${ }^{27}$ height (quintiles), annual household family income $(\leq \$ 10,000, \$ 10,001-20,000, \$ 20,001-30,000, \$ 30,001-40,000$, $\$ 40,0001-50,000, \$ 50,001-60,000, \$ 60,001-70,000$, and > $\$ 70,000$, and do not know), ethnicity (non-Hispanic white vs other), ever smoking (yes or no), ever alcohol intake (yes or no), and time of sample collection (both month of sampling for seasonal variation and hour of the day for diurnal variation in hormone levels).

Statistical Analyses. Subjects were classified as having reached puberty or not, based on self-reported menarchal status and blood sample hormone levels at the time of interview, which has been called the status quo method. ${ }^{28}$ In this analysis, pubertal status at a given age can be seen as a measure of cumulative incidence of puberty up to that age, which in turn reflects the age distribution of reaching puberty.

We estimated the association between pubertal status and concentrations of PFOA and PFOS by logistic regression, controlling for age (fitted as a cubic spline with three knots in all models separately by gender) and time of day of giving the blood sample (for boys). Age needs to be well controlled as PFC concentrations are inversely associated with age in the age range studied. $^{21,29}$ To investigate these associations and explore the shape of any exposure-response relation, we fitted each exposure variable in two ways: (i) as a categorical variable (quartiles) and (ii) as a natural log-transformed continuous variable. The natural logarithm transformation was made because, in general, in these data, associations with the exposures thus transformed were more nearly linear than those with untransformed exposures. We assessed the potential for confounding between these correlated exposures (rho $=0.27, p<0.001$ ) by inclusion of PFOA and PFOS together in models.

The median age of puberty can then be estimated from the modeled proportion reaching puberty by age, by identifying the age at which $50 \%$ prevalence was predicted. For estimating impact for each puberty indicator, we derived the shift in median age of puberty between different PFOA and PFOS categories.

We examined sensitivity of ORs for PFCs to inclusion in the model of several potentially confounding covariates, and only time of day of blood sampling in models with puberty classified using testosterone acted as a confounder, with the PFC coefficient changing by over $10 \%$ when added to the model. Information on annual household family income was available for only around two-thirds of the study population and did not act as a confounder in this subsample. Our analyses relied primarily on classification of status at interview, rather than the recalled age of menarche groups, as age was known precisely. However, as a secondary analysis, we made use of responses to the question about the broad age group of the beginning of menarche, to investigate whether in girls aged $\geq 16$ years who had started periods by the time of survey, there was evidence of later reported age at puberty according to PFC concentrations. Specifically, we consider the classification of age at first period $(<10,10-12$, $13-15$ years) as an ordered outcome and carried out an ordinal (cumulative odds) regression ${ }^{30}$ with $(\log )$ PFC concentrations. We used the statistical software package STATA for all statistical analyses (StataCorp. 2009. STATA Statistical Software: Release 11. College Station, TX: StataCorp LP). 
Table 2. Percentage of Boys and Girls Who Had Reached Puberty at Each Year of Age Based on Criteria Derived from Levels of Hormones or Self-Reported Menarche, Mid-Ohio Valley, 2005-2006

\begin{tabular}{|c|c|c|c|c|c|c|c|}
\hline age years $^{a}$ & boys $\mathrm{N}$ & $\begin{array}{l}\text { total testosterone } \\
(>50 \mathrm{ng} / \mathrm{dL}) \%\end{array}$ & $\begin{array}{l}\text { free testosterone } \\
(>5 \mathrm{pg} / \mathrm{mL}) \%\end{array}$ & girls $\mathrm{N}$ & $\begin{array}{l}\text { menarche } \\
\text { (yes) } \%\end{array}$ & $\begin{array}{c}\text { estradiol } \\
(>20 \mathrm{pg} / \mathrm{mL}) \%\end{array}$ & $\begin{array}{c}\text { estradiol } \\
\text { combined }^{b} \%\end{array}$ \\
\hline 8 & 173 & 0.00 & 0.00 & 190 & 0.54 & 14.1 & 15.0 \\
\hline 9 & 220 & 0.91 & 0.00 & 213 & 0.94 & 18.7 & 19.1 \\
\hline 10 & 244 & 4.9 & 0.00 & 226 & 5.0 & 43.5 & 44.3 \\
\hline 11 & 237 & 20.3 & 2.5 & 221 & 22.6 & 65.6 & 67.8 \\
\hline 12 & 272 & 50.4 & 10.3 & 225 & 50.9 & 86.6 & 91.4 \\
\hline 13 & 290 & 79.3 & 38.1 & 255 & 81.6 & 88.9 & 96.8 \\
\hline 14 & 299 & 94.3 & 68.2 & 291 & 96.2 & 86.9 & 99.0 \\
\hline 15 & 350 & 99.1 & 87.7 & 302 & 98.7 & 86.4 & 100 \\
\hline 16 & 334 & 100 & 93.1 & 338 & 99.4 & 83.7 & 99.7 \\
\hline 17 & 318 & 100 & 97.2 & 328 & 99.7 & 78.7 & 100 \\
\hline 18 & 339 & 100 & 97.1 & 342 & 99.7 & 71.8 & 100 \\
\hline
\end{tabular}

${ }^{a}$ Median age at which the prevalence reached 50\% per each criterion was 12.5 and 13.8 years for free and total testosterone and 12.5 and 10.8 years for menarche and estradiol combined, respectively. ${ }^{b}$ Proportion of girls either with estradiol levels $>20 \mathrm{pg} / \mathrm{mL}$ or being postmenarche.

Table 3. Association between PFOA and PFOS Concentrations and Puberty in Boys, Using Total (>50 ng/dL) and Free $(>5 \mathrm{pg} / \mathrm{mL}$ ) Testosterone Levels As Indicators, Mid-Ohio Valley, 2005-2006 ${ }^{d}$

puberty indicators

\begin{tabular}{|c|c|c|c|c|c|c|}
\hline \multirow[b]{2}{*}{ exposure } & \multicolumn{3}{|c|}{ total testosterone $(N=3072)$} & \multicolumn{3}{|c|}{ free testosterone $(N=3071)$} \\
\hline & OR & $95 \% \mathrm{CI}$ & delay $^{a}$ & OR & $95 \% \mathrm{CI}$ & delay $^{a}$ \\
\hline PFOA-Q2 & 1.01 & $0.65-1.58$ & -4 & 0.72 & $0.49-1.06$ & 76 \\
\hline PFOA-Q3 & 1.00 & $0.64-1.58$ & -1 & 0.80 & $0.54-1.18$ & 53 \\
\hline PFOA-Q4 & 0.75 & $0.49-1.15$ & 69 & 0.80 & $0.55-1.17$ & 52 \\
\hline LnPFOA & 0.87 & $0.76-0.99$ & 33 & 0.95 & $0.84-1.07$ & 13 \\
\hline $\mathrm{PFOA}-\mathrm{Q} 2^{b}$ & 1.12 & $0.71-1.75$ & -26 & 0.78 & $0.53-1.15$ & 57 \\
\hline PFOA-Q $3^{b}$ & 1.16 & $0.73-1.84$ & -35 & 0.87 & $0.59-1.30$ & 31 \\
\hline PFOA-Q4 ${ }^{b}$ & 0.88 & $0.57-1.37$ & 31 & 0.91 & $0.61-1.34$ & 23 \\
\hline $\mathrm{LnPFOA}^{b}$ & 0.91 & $0.80-1.04$ & 23 & 0.98 & $0.87-1.11$ & 4 \\
\hline PFOS-Q2 & 0.74 & $0.46-1.19$ & 70 & 0.79 & $0.53-1.18$ & 53 \\
\hline PFOS-Q3 & 0.58 & $0.37-0.90$ & 131 & 0.58 & $0.40-0.85$ & 125 \\
\hline PFOS-Q4 & 0.46 & $0.29-0.71$ & 190 & 0.57 & $0.39-0.85$ & 129 \\
\hline LnPFOS & 0.58 & $0.44-0.77$ & 130 & 0.71 & $0.54-0.92$ & 82 \\
\hline PFOS-Q2 ${ }^{c}$ & 0.74 & $0.46-1.19$ & 70 & 0.80 & $0.53-1.19$ & 52 \\
\hline $\mathrm{PFOS} \mathrm{Q}^{c}{ }^{c}$ & 0.58 & $0.37-0.91$ & 118 & 0.59 & $0.40-0.87$ & 122 \\
\hline PFOS-Q4 ${ }^{c}$ & 0.46 & $0.29-0.72$ & 187 & 0.59 & $0.39-0.88$ & 123 \\
\hline $\operatorname{LnPFOS}^{c}$ & 0.58 & $0.43-0.78$ & 129 & 0.72 & $0.55-0.95$ & 76 \\
\hline
\end{tabular}

All models adjusted for age and time of taking sample. ${ }^{a}$ Delays in median age (in days) of onset of puberty with respect to first PFOA/PFOS quartile or by unit in LnPFOA or LnPFOS. ${ }^{b}$ Models include quartiles of PFOS. ${ }^{c}$ Models include quartiles of PFOA. ${ }^{d} \mathrm{CI}=$ confidence interval; $\mathrm{OR}=$ odd ratio; $\mathrm{PFOA}=$ perfluorooctanoic acid; $\mathrm{PFOS}=$ perfluorooctane sulfonate; $\mathrm{Q}=$ quartile.

\section{RESULTS}

Study Population and PFOA and PFOS Concentration. Table 1 shows the characteristics of the study population and the PFOA and PFOS concentrations. Approximately $97 \%$ of the population was white, and $36 \%$ declared having a household family income $<\$ 20,000$ per year. Median PFOA and PFOS

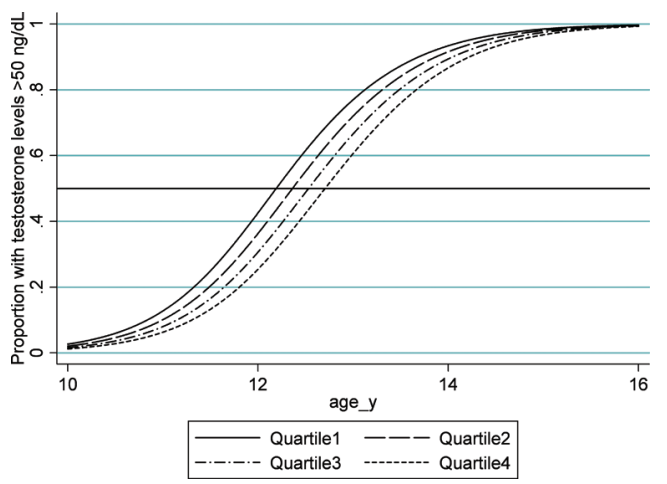

Figure 1. Proportion of boys predicted by the logistic model to reach total testosterone levels $\geq 50 \mathrm{ng} / \mathrm{dL}$ by quartiles of PFOS, Mid-Ohio Valley, 2005-2006.

concentrations were 26 and $20 \mathrm{ng} / \mathrm{mL}$ in boys and 20 and $18 \mathrm{ng} /$ $\mathrm{mL}$ in girls, respectively.

Sex Steroid Hormone Levels and Self-Reported Menarche. Table 2 shows the percentage of girls and boys who had reached puberty at each year of age, for each indicator (levels of sex steroid hormones or menarche). Transition to puberty based on these criteria was reached for most in the age range $10-15$ years, and, as expected, girls started puberty at a younger age than boys. The median age by each indicator ranged from 10.8 to 13.8 years. Increased free testosterone is a marker of a more advanced pubertal stage in boys and increased estradiol a marker of an earlier stage in girls.

Association between PFC Concentrations and Puberty at Age of Interview. Table 3 shows the association between PFC concentrations and pubertal status using raised testosterone levels in boys as the indicator. We found a clear relationship of reduced odds of having reached puberty using total testosterone (>50 ng/dL) as an indicator, with increasing PFOS (delay of 190 days between the highest and lowest quartile). For PFOA, no significant difference in risk of puberty across quartiles was found, and in the PFOA log-transformed model, the suggestion of a trend in PFOA-associated delays (puberty based on raised total testosterone) is weakened when PFOS is included. We found a similar pattern but with slightly weaker effect sizes for 
Table 4. Association between PFOA and PFOS Concentrations and Puberty in Girls, Using Menarche and Estradiol (>20 pg/mL) Levels As Indicators, Mid-Ohio Valley, 2005$2006^{e}$

puberty indicators

\begin{tabular}{|c|c|c|c|c|c|c|}
\hline \multirow[b]{3}{*}{ exposure } & \multicolumn{6}{|c|}{ puberty indicators } \\
\hline & \multicolumn{3}{|c|}{ menarche $(N=2903)$} & \multicolumn{3}{|c|}{ estradiol combined $^{a}(N=2878)$} \\
\hline & OR & $95 \% \mathrm{CI}$ & delay $^{b}$ & OR & $95 \% \mathrm{CI}$ & delay $^{b}$ \\
\hline PFOA-Q2 & 0.54 & $0.35-0.84$ & 142 & 0.68 & $0.45-1.01$ & 155 \\
\hline PFOA-Q3 & 0.50 & $0.32-0.77$ & 163 & 0.68 & $0.45-1.02$ & 158 \\
\hline PFOA-Q4 & 0.57 & $0.37-0.89$ & 130 & 0.63 & $0.43-0.93$ & 183 \\
\hline LnPFOA & 0.83 & $0.73-0.95$ & 42 & 0.89 & $0.79-1.00$ & 46 \\
\hline${\mathrm{PFOA}-\mathrm{Q} 2^{c}}^{c}$ & 0.58 & $0.37-0.90$ & 127 & 0.77 & $0.52-1.17$ & 100 \\
\hline${\mathrm{PFOA}-\mathrm{Q} 3^{c}}^{\mathrm{C}}$ & 0.55 & $0.35-0.86$ & 141 & 0.81 & $0.53-1.24$ & 82 \\
\hline PFOA-Q4 ${ }^{c}$ & 0.65 & $0.41-1.02$ & 101 & 0.82 & $0.54-1.24$ & 77 \\
\hline $\mathrm{LnPFOA}^{c}$ & 0.87 & $0.75-1.00$ & 33 & 0.96 & $0.85-1.09$ & 15 \\
\hline PFOS-Q2 & 0.72 & $0.47-1.10$ & 79 & 1.14 & $0.75-1.73$ & -55 \\
\hline PFOS-Q3 & 0.55 & $0.35-0.86$ & 141 & 0.62 & $0.41-0.93$ & 191 \\
\hline PFOS-Q4 & 0.55 & $0.35-0.87$ & 138 & 0.47 & $0.31-0.70$ & 289 \\
\hline LnPFOS & 0.60 & $0.43-0.83$ & 120 & 0.49 & $0.36-0.67$ & 289 \\
\hline${\mathrm{PFOS}-\mathrm{Q} 2^{d}}^{d}$ & 0.75 & $0.49-1.16$ & 68 & 1.12 & $0.76-1.77$ & -63 \\
\hline PFOS-Q3 ${ }^{d}$ & 0.61 & $0.38-0.96$ & 117 & 0.64 & $0.41-0.98$ & 175 \\
\hline PFOS-Q4 ${ }^{d}$ & 0.64 & $0.40-1.01$ & 105 & 0.50 & $0.33-0.76$ & 268 \\
\hline $\mathrm{LnPFOS}^{d}$ & 0.67 & $0.48-0.93$ & 94 & 0.51 & $0.37-0.71$ & 255 \\
\hline
\end{tabular}

All models adjusted for age. ${ }^{a}$ Estradiol combined indicator: either estradiol $>20 \mathrm{pg} / \mathrm{mL}$ or postmenarche. ${ }^{b}$ Delays in median age (in days) of onset of puberty with respect to first PFOA/PFOS quartile or by unit in LnPFOA or LnPFOS. ${ }^{c}$ Models include quartiles of PFOS. ${ }^{d}$ Models include quartiles of PFOA. ${ }^{e} \mathrm{CI}=$ confidence interval; $\mathrm{OR}=$ odd ratio; $\mathrm{PFOA}$ = perfluorooctanoic acid; PFOS = perfluorooctane sulfonate; $\mathrm{Q}=$ quartile.

free testosterone ( $>5 \mathrm{pg} / \mathrm{mL})$. Figure 1 illustrates the model fit and the shift (in relation to PFOS quartiles) in the distribution of the prevalence by age of having reached puberty by using the indicator of testosterone $>50 \mathrm{ng} / \mathrm{dL}$. The age at which each line reaches $50 \%$ is the median age of achieving puberty for that group, and the displacement to older ages with increasing quartile of PFOS is the difference between these predicted median ages.

Table 4 shows the association between PFC concentrations and pubertal status in girls using self-reported menarche and the estradiol combined indicator (either estradiol levels $>20 \mathrm{pg} / \mathrm{mL}$ or report menarche). Higher concentrations of PFOA or PFOS were associated with reduced odds of postmenarche (130 and 138 days of delay between the highest and lowest quartile, respectively). In models containing both PFCs, there was still evidence of an effect of each. For puberty derived from estradiol combined indicator, there was evidence of an association with PFOS but not PFOA, with a delay of 289 days between first and fourth quartile of PFOS.

Sensitivity analyses were conducted to investigate if our model results were affected by inclusion of other variables (month of sampling, race, BMI, height, annual household family income, smoking, or alcohol intake) and which, apart from BMI and height, did not show any changes in the puberty-PFC associations. In models including BMI (which shows a strong association with puberty), the ORs for total testosterone models were $0.86(95 \% \mathrm{CI}=0.76-0.99)$ and $0.49(95 \% \mathrm{CI}=0.36-0.67)$ for log-transformed PFOA and PFOS concentrations, respectively, and ORs in menarche models were 0.84 (95\% CI $=0.73-0.97)$ and 0.76 (95\% CI $=0.55-1.05$ ), for log PFOA and PFOS, respectively. In models including height (which also shows a strong association with puberty), the ORs for total testosterone models were $0.87(95 \% \mathrm{CI}=0.76-0.99)$ and $0.60(95 \% \mathrm{CI}=$ 0.44-0.80) for log PFOA and PFOS concentrations, respectively, and ORs in menarche models were 0.83 (95\% CI = $0.72-0.96)$ and $0.73(95 \% \mathrm{CI}=0.52-1.01)$, for $\log$ PFOA and PFOS, respectively. All results for PFOA were virtually identical, and for PFOS there were modest shifts; for example, for menarche, where including BMI or height changed the OR from 0.60 , without these terms, to 0.76 or 0.73 . From these sensitivity analyses, neither height nor BMI was judged to be a strong confounder; however, even these changes in OR need to be treated with caution and their inclusion may lead to an overadjustment. Insofar as height or BMI change is a consequence of pubertal maturation, any association between PFC and these attributes may be mediated by puberty, the outcome of interest.

Respondents had provided information on age of menarche based on recall, and these were examined in a secondary analysis for consistency with our findings. Among girls $\geq 16$ years the OR from ordinal regression across the three age groupings $(<10$, $10-12,13-15$ years) indicated a reduced risk of younger menarche in association with PFOA, OR 0.83 per log unit PFOA (95\% CI $=0.74-0.93)$ but not in relation to PFOS, OR 0.87 (0.69-1.09). These findings are consistent in direction with the main analysis results.

\section{DISCUSSION}

In the present cross-sectional analysis, PFOA and PFOS concentrations were associated with later age of sexual maturation to various degrees, in boys and girls, as measured by sex steroid hormones and by self-reported age of menarche, as indicators of having reached puberty. For PFOS, associations were found with later age of having reached puberty in boys and girls using each of the indicators of puberty. For PFOA, the evidence for later puberty was evident for girls but not for boys.

To our knowledge, there is no previous epidemiological study on exposure to these contaminants and sexual development in boys to compare our results of later sexual maturation associated with PFOS concentrations (190 days between the highest and lowest quartile). In adults, cross sectional studies of PFOA/ PFOS concentrations and testosterone levels found no associations in a study among employees with occupational exposure ${ }^{14}$ or in the Danish general population. ${ }^{15}$

In girls, our results on an association between concentrations of PFOA or PFOS and later age at having reached puberty (130 and 138 days, respectively) can be compared with two studies. Christensen et al. ${ }^{17}$ assessed puberty and PFCs in the UKALSPAC birth cohort. They compared 218 girls with early puberty (reported as <11.5 years) with a similar number with later puberty, in relation to PFC concentrations in cord blood. Exposure contrasts for PFOS were similar to the present study (interquartile range $=15-25$ versus $14-27 \mathrm{ng} / \mathrm{mL}$ in the present study) but much lower for PFOA (2.8-4.8 versus $11-58 \mathrm{ng} / \mathrm{mL}$ in this study). They computed the ORs for early puberty in relation to log-transformed PFC concentrations or dichotomized exposures and in no case did the confidence intervals exclude 1.0. Of the ten PFCs reported, the strongest effect in terms of adjusted ORs per unit change in (natural) log PFC was for PFOS with a value of $0.68(95 \% \mathrm{CI}=0.40-1.13)$ 
of a similar magnitude to our result $(\mathrm{OR}=0.60,95 \% \mathrm{CI}=$ $0.43-0.83$ ). For PFOA, their adjusted OR is close to 1 , with wide confidence intervals $(\mathrm{OR}=1.01,95 \% \mathrm{CI}=0.61-1.68)$ per unit change in natural log PFOA concentration. ${ }^{17} \mathrm{~A}$ positive relationship between higher PFOA concentrations and Tanner stage $\mathrm{B} 2+$ of breast maturation at the 1 year follow-up visit was reported in a study in Ohio, USA, suggesting earlier puberty in relation to PFOA concentrations. ${ }^{18}$ The differences between these two last epidemiological studies and the present one include the following: the population size, the specific outcome considered or measurement of it, exposure concentrations (much higher PFOA concentrations in the present population), and the type of study (cross-sectional versus prospective in the previous two studies).

Puberty is a vulnerable stage of life where disturbance has been linked to increased health problems. Thus, severe delays in the onset of puberty ( $>18$ years) in girls have been reported to be a risk factor for infertility, ${ }^{31}$ and more modest pubertal delays ( $\geq 13$ years) have been associated with endometriosis, which may be in the pathway for infertility. ${ }^{32}$ The parallel finding of delay in puberty among boys is less clearly associated with reproductive health problems. Delays in puberty have been associated with higher risk of osteopenia in adult men ${ }^{33}$ and with bone mineral density in adult women ${ }^{34}$ which may be related with osteoporosis. Delayed puberty has also been associated with psychosocial problems. ${ }^{35}$ Our findings of statistically significant delays in the age of having reached puberty are of interest, although whether a later age in sexual maturation of 3 to 6 months has clinically important long-term impacts on physical health and psychological wellbeing has yet to be determined.

The strengths of the present study include the large sample size and the fact that the participation rate in the community was high, diminishing concern about potential selection biases and chance findings. It is believed that this population is representative of all those children who drank contaminated water in the Mid-Ohio Valley. The age range studied is relatively narrow, and these compounds have long half-lives; therefore, unless puberty itself or related phenomena changed concentrations of PFCs in serum then a good level of correlation between exposure at the time of survey and only a few years earlier would be expected. It is reassuring for the validity of our data that the pattern of increase in prevalence of menarche according to age is broadly in accordance with that reported by a study of 18,549 girls surveyed in 1992-1993 in the US. ${ }^{36}$ Mean (SD) age of menarche in that study was 12.9 (1.2) years versus a median age of 12.5 years in the present study. The availability of time of day of sampling enabled us to successfully adjust for time to avoid potential confounding from diurnal fluctuations in testosterone levels. Finally, monitoring of hormone levels is emerging as a useful marker of sexual maturation, and the selected hormone cutoff points in the present study correlated with classifications by Tanner stages in previous reports. ${ }^{23,25,37,38}$

A weakness of the present study is the lack of PFC concentrations measures prior to puberty. For those who had reached puberty, the measure of PFCs in blood was taken after puberty had been reached, and there is a concern that behavioral and physiologic changes associated with puberty (including menstrual blood loss) may lead to changes in PFC concentrations in serum, and for PFOA in particular, a change in water consumption patterns and hence uptake. For example, if puberty causes changes in PFOA and PFOS blood concentrations due to menstrual blood loss, this could manifest itself as menarche appearing to be inversely associated with exposure. Further work on this population in relation to PFOA concentrations, before puberty starts, will be able to address some of these concerns. A further limitation is the lack of direct information on secondary sexual maturation, for example, according to Tanner's criteria. ${ }^{39,40}$ Use of Tanner's criteria is not straightforward due to possible interobserver variability. A combination of physical examination and biomarker measures would be desirable.

In conclusion, these cross-sectional analyses suggest later age of pubertal maturation, using sex hormone levels as markers of sexual development, is correlated with PFOS in boys and later age of menarche is correlated with PFOA and PFOS concentrations in girls. Our results suggest later age at having reached puberty of 3 to 6 months across the range of concentrations found in this population for both boys and girls.

\section{AUTHOR INFORMATION}

\section{Corresponding Author}

*Phone: +44 207927 2066. Fax: +44 207927 2701. E-mail: Maria-Jose.Lopez@lshtm.ac.uk.

\section{ACKNOWLEDGMENT}

We thank the participants for their contributions to this study. This work was funded by the C8 Class Action Settlement Agreement (Circuit Court of Wood County, WV, USA) between DuPont and plaintiffs, which resulted from releases of perfluorooctanoic acid (PFOA or C8) into drinking water. It is part of the research undertaken by the Court-approved C8 Science Panel established under the same Settlement Agreement. The task of the C8 Science Panel, of which Tony Fletcher is a member, is to undertake research in the Mid-Ohio Valley in order to evaluate the results and other available information to determine if there are any probable links between PFOA and disease. Funds were administered by the Garden City Group (Melville, NY) that reports to the Court. The authors of this manuscript declare that their ability to design, conduct, interpret, or publish research was unimpeded by and fully independent of the court and/or settling parties. In addition, they declare no competing financial interests. We are grateful for the helpful comments on the manuscript from D. Savitz, K. Steenland, and A. Winquist; information on classifying contraceptive hormone use from B. Javins and S. Knox; and manuscript editing from A. Beierholm. The LSHTM Ethics Committee approved this study.

\section{REFERENCES}

(1) Lau, C.; Anitole, K.; Hodes, C.; Lai, D.; Pfahles-Hutchens, A.; Seed, J. Perfluoroalkyl acids: a review of monitoring and toxicological findings. Toxicol. Sci. 2007, 99 (2), 366-94.

(2) Steenland, K.; Fletcher, T.; Savitz, D. A. Epidemiologic evidence on the health effects of perfluorooctanoic acid (PFOA). Environ. Health Perspect. 2010, 118 (8), 1100-8.

(3) Jensen, A. A.; Leffers, H. Emerging endocrine disrupters: perfluoroalkylated substances. Int. J. Androl. 2008, 31 (2), 161-9.

(4) Biegel, L. B.; Liu, R. C.; Hurtt, M. E.; Cook, J. C. Effects of ammonium perfluorooctanoate on Leydig cell function: in vitro, in vivo, and ex vivo studies. Toxicol. Appl. Pharmacol. 1995, 134 (1), 18-25.

(5) Cook, J. C.; Murray, S. M.; Frame, S. R.; Hurtt, M. E. Induction of Leydig cell adenomas by ammonium perfluorooctanoate: a possible endocrine-related mechanism. Toxicol. Appl. Pharmacol. 1992, 113 (2), 209-17.

(6) Zhao, Y.; Tan, Y. S.; Haslam, S. Z.; Yang, C. Perfluorooctanoic acid effects on steroid hormone and growth factor levels mediate 
stimulation of peripubertal mammary gland development in C57BL/6 mice. Toxicol. Sci. 2010, 115 (1), 214-24.

(7) Seacat, A.; Thomford, P.; Hansen, K.; Olsen, G.; Case, M.; Butenhoff, J. Subchronic toxicity studies on perfluorooctanesulfonate potassium salt in cynomolgus monkeys. Toxicol. Sci. 2002, 68 (1).

(8) Oakes, K. D.; Sibley, P. K.; Martin, J. W.; MacLean, D. D.; Solomon, K. R.; Mabury, S. A.; Van Der Kraak, G. J. Short-term exposures of fish to perfluorooctane sulfonate: acute effects on fatty acyl-coa oxidase activity, oxidative stress, and circulating sex steroids. Environ. Toxicol. Chem. 2005, 24 (5), 1172-81.

(9) Lau, C.; Thibodeaux, J. R.; Hanson, R. G.; Rogers, J. M.; Grey, B. E.; Stanton, M. E.; Butenhoff, J. L.; Stevenson, L. A. Exposure to perfluorooctane sulfonate during pregnancy in rat and mouse. II: postnatal evaluation. Toxicol. Sci. 2003, 74 (2), 382-92.

(10) Butenhoff, J. L.; Kennedy, G. L., Jr.; Frame, S. R.; O’Connor, J. C.; York, R. G. The reproductive toxicology of ammonium perfluorooctanoate (APFO) in the rat. Toxicology 2004, 196 (1-2), 95-116.

(11) White, S. S.; Calafat, A. M.; Kuklenyik, Z.; Villanueva, L.; Zehr, R. D.; Helfant, L.; Strynar, M. J.; Lindstrom, A. B.; Thibodeaux, J. R.; Wood, C.; Fenton, S. E. Gestational PFOA exposure of mice is associated with altered mammary gland development in dams and female offspring. Toxicol. Sci. 2007, 96 (1), 133-44.

(12) Yang, C.; Tan, Y. S.; Harkema, J. R.; Haslam, S. Z. Differential effects of peripubertal exposure to perfluorooctanoic acid on mammary gland development in $\mathrm{C} 57 \mathrm{Bl} / 6$ and $\mathrm{Balb} / \mathrm{c}$ mouse strains. Reprod. Toxicol. 2009, 27 (3-4), 299-306.

(13) Lau, C.; Thibodeaux, J. R.; Hanson, R. G.; Narotsky, M. G.; Rogers, J. M.; Lindstrom, A. B.; Strynar, M. J. Effects of perfluorooctanoic acid exposure during pregnancy in the mouse. Toxicol. Sci. 2006, 90 (2), 510-8.

(14) Olsen, G. W.; Gilliland, F. D.; Burlew, M. M.; Burris, J. M.; Mandel, J. S.; Mandel, J. H. An epidemiologic investigation of reproductive hormones in men with occupational exposure to perfluorooctanoic acid. J. Occup. Environ. Med. 1998, 40 (7), 614-22.

(15) Joensen, U. N.; Bossi, R.; Leffers, H.; Jensen, A. A.; Skakkebaek, N. E.; Jorgensen, N. Do perfluoroalkyl compounds impair human semen quality? Environ. Health Perspect. 2009, 117 (6), 923-7.

(16) Knox, S. S.; Jackson, T.; Javins, B.; Frisbee, S. J.; Shankar, A.; Ducatman, A. M. Implications of early menopause in women exposed to perfluorocarbons. J. Clin. Endocrinol. Metab. 2011 doi:10.1210/jc.20102401.

(17) Christensen, K. Y.; Maisonet, M.; Rubin, C.; Holmes, A.; Calafat, A. M.; Kato, K.; Flanders, W. D.; Heron, J.; McGeehin, M. A.; Marcus, M. Exposure to polyfluoroalkyl chemicals during pregnancy is not associated with offspring age at menarche in a contemporary British cohort. Environ. Int. 2010.

(18) Pinney, S. M.; Windham, G. C.; Biro, F. M.; Kushi, L. H.; Yaghjyan, L.; Calafat, A. M.; Kato, K.; Succop, P.; Brown, M. K.; Hernick, A.; Bornschein, R. Perfluorooctanoic acid (PFOA) and pubertal maturation in young girls. Abstract- ISEE 21st annual conference, Dublin, Ireland, August 25-29, 2009. Published in: Epidemiology 2009, 20 (6), S80.

(19) Maras, M.; Vanparys, C.; Muylle, F.; Robbens, J.; Berger, U.; Barber, J. L.; Blust, R.; De Coen, W. Estrogen-like properties of fluorotelomer alcohols as revealed by mcf-7 breast cancer cell proliferation. Environ. Health Perspect. 2006, 114 (1), 100-5.

(20) Liu, C.; Du, Y.; Zhou, B. Evaluation of estrogenic activities and mechanism of action of perfluorinated chemicals determined by vitellogenin induction in primary cultured tilapia hepatocytes. Aquat. Toxicol. 2007, 85 (4), 267-77.

(21) Frisbee, S. J.; Brooks, A. P., Jr.; Maher, A.; Flensborg, P.; Arnold, S.; Fletcher, T.; Steenland, K.; Shankar, A.; Knox, S. S.; Pollard, C.; Halverson, J. A.; Vieira, V. M.; Jin, C.; Leyden, K. M.; Ducatman, A. M. The C8 Health Project: design, methods, and participants. Environ. Health Perspect. 2009, 117 (12), 1873-82.

(22) Centers for Disease Control and Prevention (CDC). Fourth National Report on Human Exposure to Environmental ChemicalsUpdated Tables. http://www.cdc.gov/exposurereport/ (accessed on $15 / 03 / 2011)$.
(23) Rapkin, A.; Tsao, J.; Turk, N.; Anderson, M.; Zeltzer, L. Relationships among self-rated Tanner staging, hormones, and psychosocial factors in healthy female adolescents. J. Pediatr. Adol. Gynecol. 2006, 19 (3), 181-187.

(24) Apter, D. Serum steroids and pituitary hormones in female puberty: a partly longitudinal study. Clin. Endocrinol. 1980, 12 (2), 107-120.

(25) Garcia-Mayor, R. V.; Andrade, M. A.; Rios, M.; Lage, M.; Dieguez, C.; Casanueva, F. F. Serum leptin levels in normal children: relationship to age, gender, body mass index, pituitary-gonadal hormones, and pubertal stage. J. Clin. Endocrinol. Metab. 1997, 82 (9), 2849-55.

(26) Santelli, J. S.; Lindberg, L. D.; Finer, L. B.; Singh, S. Explaining recent declines in adolescent pregnancy in the United States: the contribution of abstinence and improved contraceptive use. Am. J. Public Health 2007, 97 (1), 150-6.

(27) CDC Epi Info. http://www.cdc.gov/epiinfo (accessed on 12/ 03/2011).

(28) Denham, M.; Schell, L. M.; Deane, G.; Gallo, M. V.; Ravenscroft, J.; DeCaprio, A. P. Relationship of lead, mercury, mirex, dichlorodiphenyldichloroethylene, hexachlorobenzene, and polychlorinated biphenyls to timing of menarche among Akwesasne Mohawk girls. Pediatrics 2005, 115 (2), e127-34.

(29) Steenland, K.; Jin, C.; MacNeil, J.; Lally, C.; Ducatman, A.; Vieira, V.; Fletcher, T. Predictors of PFOA levels in a community surrounding a chemical plant. Environ. Health Perspect. 2009, 117 (7), 1083-8.

(30) Armstrong, B. G.; Sloan, M. Ordinal regression models for epidemiologic data. Am. J. Epidemiol. 1989, 129 (1), 191-204.

(31) Komura, H.; Miyake, A.; Chen, C. F.; Tanizawa, O.; Yoshikawa, $\mathrm{H}$. Relationship of age at menarche and subsequent fertility. Eur. J. Obstet. Gynecol. Reprod. Biol. 1992, 44 (3), 201-3.

(32) Berube, S.; Marcoux, S.; Maheux, R. Characteristics related to the prevalence of minimal or mild endometriosis in infertile women. Canadian Collaborative Group on Endometriosis. Epidemiology 1998, 9 (5), 504-10

(33) Finkelstein, J. S.; Neer, R. M.; Biller, B. M.; Crawford, J. D.; Klibanski, A. Osteopenia in men with a history of delayed puberty. $N$ Engl. J. Med. 1992, 326 (9), 600-4.

(34) Chevalley, T.; Bonjour, J. P.; Ferrari, S.; Rizzoli, R. Influence of age at menarche on forearm bone microstructure in healthy young women. J. Clin. Endocrinol. Metab. 2008, 93 (7), 2594-601.

(35) Patton, G. C.; Viner, R. Pubertal transitions in health. Lancet 2007, 369 (9567), 1130-9.

(36) Herman-Giddens, M. E.; Slora, E. J.; Wasserman, R. C.; Bourdony, C. J.; Bhapkar, M. V.; Koch, G. G.; Hasemeier, C. M. Secondary sexual characteristics and menses in young girls seen in office practice: a study from the Pediatric Research in Office Settings network. Pediatrics 1997, 99 (4), 505-12.

(37) Raman, A.; Lustig, R.; Fitch, M.; Fleming, S. Accuracy of self assessed Tanner staging against hormonal assessment of sexual maturation in overweight African-American children. J. Pediatr. Endocrinol. Metab. 2009, 22, 609-622.

(38) Schoeters, G.; Den Hond, E.; Dhooge, W.; van Larebeke, N.; Leijs, M. Endocrine disruptors and abnormalities of pubertal development. Basic Clin. Pharmacol. Toxicol. 2008, 102 (2), 168-75.

(39) Tanner, J. M. Growth at Adolescence, 2nd ed.; Blackwell: Oxford, 1962.

(40) Tanner, J. M. Growth and Endocrinology in the adolescent. In Endocrine and Genetic Diseases of Childhood; Gardner, L., Eds.; Saunders WB: Philadelphia, 1969. 